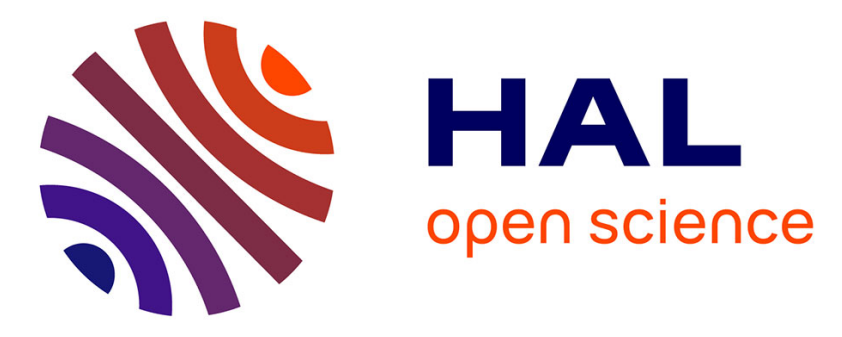

\title{
Blind Channel Estimation for STBC Systems Using Higher-Order Statistics
}

Vincent V. Choqueuse, Ali Mansour, Gilles Burel, Ludovic Collin, Koffi Clément Yao

\section{- To cite this version:}

Vincent V. Choqueuse, Ali Mansour, Gilles Burel, Ludovic Collin, Koffi Clément Yao. Blind Channel Estimation for STBC Systems Using Higher-Order Statistics. IEEE Transactions on Wireless Communications, 2011, 10 (2), pp.495 - 505. 10.1109/TWC.2010.112310.091576 . hal-00566506

\section{HAL Id: hal-00566506 https://hal.science/hal-00566506}

Submitted on 16 Feb 2011

HAL is a multi-disciplinary open access archive for the deposit and dissemination of scientific research documents, whether they are published or not. The documents may come from teaching and research institutions in France or abroad, or from public or private research centers.
L'archive ouverte pluridisciplinaire HAL, est destinée au dépôt et à la diffusion de documents scientifiques de niveau recherche, publiés ou non, émanant des établissements d'enseignement et de recherche français ou étrangers, des laboratoires publics ou privés. 


\title{
Blind Channel Estimation for STBC Systems Using Higher-Order Statistics
}

\author{
Vincent Choqueuse, Member, IEEE, Ali Mansour, Senior Member, IEEE, Gilles Burel, Senior Member, IEEE, \\ Ludovic Collin, and Koffi Yao, Member, IEEE
}

\begin{abstract}
This paper describes a new blind channel estimation algorithm for Space-Time Block Coded (STBC) systems. The proposed method exploits the statistical independence of sources before space-time encoding. The channel matrix is estimated by minimizing a kurtosis-based cost function after Zero-Forcing equalization. In contrast to subspace or Second-Order Statistics (SOS) approaches, the proposed method is more general since it can be employed for the general class of linear STBCs including Spatial Multiplexing, Orthogonal, quasi-Orthogonal and NonOrthogonal STBCs. Furthermore, unlike other approaches, the method does not require any modification of the transmitter and, consequently, is well-suited for non-cooperative context. $\mathrm{Nu}$ merical examples corroborate the performance of the proposed algorithm.
\end{abstract}

Index Terms-MIMO, space time coding, channel estimation, independent component analysis, higher-order statistics.

\section{INTRODUCTION}

$\mathbf{S}$ PACE-TIME Block Coding is a set of practical signal design techniques aimed at approaching the information theoretic capacity limit of Multiple-Input Multiple-Output (MIMO) channels. Since the pioneer work of Alamouti [1], space-time coding has been a fast growing field of research. In the last decade, numerous coding schemes have been proposed. These include orthogonal (OSTBCs) [1]-[3], quasiorthogonal (QOSTBCs) [4], [5] and non-orthogonal STBCs (NOSTBCs) [6]. At the receiver side, the decoding is achieved by a space-time equalizer. Most space-time equalizers require Channel State Information (CSI). This information is usually obtained through training based techniques at the expense of the bandwidth efficiency. On the other hand, the differential schemes proposed in [7]-[10], which do not require CSI, incur a penalty in performance of at least $3 \mathrm{~dB}$ as compared to the coherent Maximum-Likelihood (ML) receiver. The drawbacks of training-based approaches and differential schemes have motivated an increasing interest in the development of blind

Manuscript received October 23, 2009; revised March 2, 2010 and October 1, 2010; accepted October 18, 2010. The associate editor coordinating the review of this paper and approving it for publication was L. Deneire.

V. Choqueuse was with the Lab-STICC, UEB, Universite de Brest; UMR CNRS 3192, CS 93837, 29238 Brest cedex 3, France. He is now with LBMS, EA 4325, same address (e-mail: vincent.choqueuse@ gmail.com).

A. Mansour is with the Department of Electrical and Computer Engineering, Curtin University, Perth, Australia (e-mail: ali.mansour@ieee.org).

G. Burel, L. Collin, and K. Yao are with the Lab-STICC, UEB, Université de Brest; UMR CNRS 3192, CS 93837, 29238 Brest cedex 3, France (e-mail: \{Gilles.Burel, Ludovic.Collin, Koffi-Clement.Yao\}@univ-brest.fr).

The authors would like to thank Janet Leschaeve for her help in checking English usage, as well as the associate editor and the anonymous reviewers for their useful comments.

Digital Object Identifier 10.1109/TWC.2010.112310.091576 channel estimation algorithms for STBC systems. Development of blind receivers also has applications in military communication system when the transmitted symbols have to be estimated in a blind fashion.

Blind channel estimation algorithms based on MaximumLikelihood (ML) have been proposed in [11], [12]. Despite their high performances, the computational costs of the MLbased methods become prohibitive for high-order modulations. In the case of BPSK or QPSK constellations, the blind-ML detection can be simplified to a Boolean Quadratic Program (BQP) [13]. For more general settings, iterative procedure can be employed to avoid the computational complexity of the ML approach. These include the Cyclic ML [12] and the Expectation-Maximisation (EM) [14], [15] algorithms. However, these iterative methods require a careful initialization of the channel and/or symbols. In particular, a poor initialization can strongly affect the Symbol-Error Rate (SER) performance.

To avoid the drawbacks of ML-based channel estimation algorithms, several authors have investigated the use of subspace [16], [17] or Second-Order Statistics (SOS) [18]-[21] approaches. However, excluding some specific low-rate codes, these approaches fail to extract the channel in a full-blind context [16]-[22]. Several approach have been proposed in literature to solve this problem, including the transmission of a short training sequence [16], [17] or the use of precoders [18], [20], [21]. However, these semi-blind methods cannot be employed in a non-cooperative scenario since they require modification of the transmitter.

One solution to avoid the limitations of SOS and subspace algorithms is to exploit Higher-Order Statistics (HOS). This approach is usually called Independent Component Analysis (ICA) [23]. ICA was originally developed for non-coded systems. Recently several authors have investigated its extension to STBC communications [24]-[31]. Nevertheless, these extensions have several limitations and drawbacks. In particular, the algorithms [24]-[29] are limited to a sub-class of Orthogonal STBCs and their extension to the general class of STBCs is far from trivial. On the other hand, the methods [30], [31] do not take into account the specific structure of the STBC.

Despite this rich literature, none of the previous algorithms is able to estimate the channel matrix for general STBCs without modification of the transmitter (pilot sequence, precoding). In this paper, an original algorithm is proposed which is wellsuited to the general class of linear STBCs whatever the coderate and/or the modulation. The channel matrix is estimated 
by minimizing a kurtosis-based cost function. In contrast with classical ICA algorithms, the cost-function is computed from the Zero-Forcing (ZF) space-time equalized symbols. Our proposed method has low-complexity and does not require any channel initialization, the use of pilot sequence and/or knowledge of modulation. Furthermore, our method does not require any modification of the transmitter and, consequently, can be employed in a non-cooperative scenario.

This paper is organized as follows. The signal models and the assumptions are presented in section II. The kurtosis-based cost function is described in section III and the minimization algorithm is described in section IV. The set of the remaining ambiguities after channel estimation is provided in section $\mathrm{V}$. Finally, the simulation results are presented in section VI.

\section{Signal Models AND Assumptions}

Hereinafter, bold upper case letters denote matrices, e.g., $\mathbf{X}$; bold lower case letters stand for column vectors, e.g., $\mathbf{x}$, and lower case letters represent scalars. Superscripts $(\cdot)^{\mathrm{T}}$ and $(\cdot)^{\mathrm{H}}$ denote transpose and Hermitian, respectively. Symbol $j=\sqrt{-1}$ is the imaginary unit, $(\cdot)^{*}$ corresponds to the complex conjugate and the operators $\Re e(\cdot)$ and $\Im m(\cdot)$ denote the real and imaginary parts, respectively. The symbol $\otimes$ is the Kronecker product, trace $(\cdot)$ is the trace function and $\mathcal{E}[\cdot]$ is the expectation operator. The $n \times n$ matrices $\mathbf{I}_{n}$ and $\mathbf{0}_{n}$ correspond to the identity and zero matrices, respectively. The unit vector, $\mathbf{e}_{k}^{(n)}$, is an $n$-dimensional row vector with " $1 "$ in its $k^{\text {th }}$ component and zero elsewhere i.e.

$$
\mathbf{e}_{k}^{(n)}=\left[\begin{array}{lllllll}
0 & k^{t h} \\
& \cdots & 0 & 1 & 0 & \cdots & 0
\end{array}\right]
$$

The elementary matrix, $\mathbf{E}_{u v}^{(n)}$, is an $n \times n$ matrix which is "1" in the $u^{t h}$ row and $v^{t h}$ column and zero elsewhere i.e.

$$
\mathbf{E}_{u v}^{(n)}=\left[\begin{array}{ccccc} 
& v^{t h} & \\
& \downarrow & & \\
& \vdots & & \\
& & 0 & & \\
\cdots & 0 & 1 & 0 & \cdots \\
& & 0 & & \\
& & \vdots & &
\end{array}\right] \leftarrow u^{t h}
$$

\section{A. Transmitted signal model}

Let us consider a linear STBC that transmits $n$ symbols during $l$ time slots through $n_{t}$ antennae. The space-time block encoder generates an $n_{t} \times l$ block matrix from a block of $n$ symbols $\mathbf{s}=\left[s_{1}, \cdots, s_{n}\right]^{\mathrm{T}}$. The block matrix, $\mathcal{C}(\mathbf{s})$, can be expressed under the general form [32]

$$
\mathcal{C}(\mathbf{s})=\sum_{k=1}^{n}\left(\mathbf{A}_{k} \Re e\left(s_{k}\right)+\mathbf{A}_{k+n} \Im m\left(s_{k}\right)\right)
$$

where the $n_{t} \times l$ matrices $\mathbf{A}_{k}$ are the space-time coding matrices.

\section{B. Signal model of received samples}

Let us consider a receiver composed of $n_{r}$ antennae. Let us also assume a quasi-static frequency-flat channel modelled by an $n_{r} \times n_{t}$ complex matrix $\mathbf{H}$. The $v^{t h}$ received block, denoted by the $n_{r} \times l$ matrix $\mathbf{Y}_{v}$, is given by [32]

$$
\mathbf{Y}_{v}=\mathbf{H C}\left(\mathbf{s}_{v}\right)+\mathbf{B}_{v}
$$

where the $n_{r} \times l$ matrix $\mathbf{B}_{v}=\left[\mathbf{b}_{v}(1), \cdots, \mathbf{b}_{v}(l)\right]$ refers to the additive noise and $\mathbf{b}_{v}(u)$ is a $n_{r}$-dimensional column vector. The aim of this study is to estimate $\mathbf{H}$ from the received data blocks, $\mathbf{Y}_{v}$, under the following assumptions:

- AS1) the $n_{r} \times n_{t}$ channel matrix, $\mathbf{H}$, is of full-column rank. Furthermore, the number of receiver antennae is strictly greater than the number of transmitters, i.e. $n_{r}>$ $n_{t}$.

- AS2) the noise vector is both spatially and temporally white with a variance of $\sigma^{2}$ per complex dimension. In particular, it implies that:

$$
\mathcal{E}\left[\mathbf{B}_{v} \mathbf{B}_{v}^{\mathrm{H}}\right]=\sigma^{2} l \mathbf{I}_{n_{r}}
$$

- AS3) the transmitted symbols, $\mathbf{s}_{v}$, are non gaussian, independent and identically distributed (i.i.d).

- AS4) the average transmit power on each antenna is normalized to unity which also implies that:

$$
\mathcal{E}\left[\mathcal{C}\left(\mathbf{s}_{v}\right) \mathcal{C}^{\mathrm{H}}\left(\mathbf{s}_{v}\right)\right]=l \mathbf{I}_{n_{t}} .
$$

- AS5) the space-time code is known at the receiver side.

Assumptions AS1), AS2) and AS3) are widely used and AS4) is respected for most STBCs ${ }^{1}$. Moreover in many scenarios, the space-time code is usually assumed to be known, otherwise, it can be estimated with a blind STBC recognition algorithm [33]-[36]. It should be noted that condition AS5) also implies that $n, l, n_{t}$ and $\mathbf{A}_{k}$ are known at the receiver side.

\section{Channel Estimation Strategy}

In this section, a new blind channel estimation strategy based on HOS is proposed. The method is composed of two steps which are detailed in the subsections III-A and III-B, respectively.

\section{A. Step 1: Data Whitening}

In the preprocessing step, the channel is estimated up to a unitary matrix through the use of SOS. By using assumptions AS1), AS2), AS3) and AS4), the $n_{r} \times n_{r}$ covariance matrix of the noiseless transmitted signals $\mathbf{R}=\mathcal{E}\left[\mathbf{Y}_{v} \mathbf{Y}_{v}^{\mathrm{H}}\right]-l \sigma^{2} \mathbf{I}_{n_{r}}$ can be expressed as

$$
\begin{aligned}
\mathbf{R} & =\mathbf{H} \mathcal{E}\left[\mathcal{C}\left(\mathbf{s}_{v}\right) \mathcal{C}^{\mathrm{H}}\left(\mathbf{s}_{v}\right)\right] \mathbf{H}^{\mathrm{H}} \\
& =l \mathbf{H} \mathbf{H}^{\mathrm{H}} .
\end{aligned}
$$

Under assumption AS1), the rank of the symmetric matrix $\mathbf{R}$ is equal to $n_{t}$. Therefore, $\mathbf{R}$ can be decomposed as follows:

\footnotetext{
${ }^{1}$ It should be noted that if $\mathcal{E}\left[\mathcal{C}\left(\mathbf{s}_{v}\right) \mathcal{C}^{\mathrm{H}}\left(\mathbf{s}_{v}\right)\right]=\alpha l \mathbf{I}_{n_{t}}$, the scaling factor $\alpha$ can be absorbed into the channel matrix $\mathbf{H}$ without loss of generality
} 
$\mathbf{R}=\mathbf{U} \Lambda \mathbf{U}^{\mathrm{H}}$, where $\mathbf{U}$ is an $n_{r} \times n_{t}$ matrix satisfying $\mathbf{U}^{\mathrm{H}} \mathbf{U}=$ $\mathbf{I}_{n_{t}}$ and $\Lambda$ is an $n_{t} \times n_{t}$ diagonal matrix containing real entries. From (7), it follows that the channel matrix $\mathbf{H}$ can be expressed as

$$
\mathbf{H}=\frac{1}{\sqrt{l}} \mathbf{U} \Lambda^{\frac{1}{2}} \mathbf{W}^{\mathrm{H}}
$$

where $\mathbf{W}$ is an $n_{t} \times n_{t}$ full rank unitary matrix.

After the preprocessing step, the determination of the matrix $\mathbf{H}$ reduces to the determination of the $n_{t} \times n_{t}$ unitary matrix $\mathbf{W}$. To determine $\mathbf{W}$, let us define the $n_{t} \times l$ whitened data block, $\mathbf{X}_{v}$, as

$$
\mathbf{X}_{v}=\sqrt{l} \Lambda^{-\frac{1}{2}} \mathbf{U}^{\mathrm{H}} \mathbf{Y}_{v}
$$

\section{B. Determining the unitary matrix $\mathbf{W}$}

1) The Zero-Forcing receiver: In this paragraph, a linear Zero-Forcing (ZF) decoder is expressed in terms of the $n_{t} \times l$ whitened data block $\mathbf{X}_{v}$. Using (4) and (8), it can be shown that

$$
\mathbf{X}_{v}=\mathbf{W}^{\mathrm{H}} \mathcal{C}\left(\mathbf{s}_{v}\right)+\underbrace{\sqrt{l} \Lambda^{-\frac{1}{2}} \mathbf{U}^{\mathrm{H}} \mathbf{B}_{v}}_{\mathbf{N}_{v}}
$$

where the $n_{t} \times l$ matrix $\mathbf{N}_{v}$ is a multidimensional zero-mean Gaussian signal. Let us define, $\tilde{\mathbf{s}}_{v}$, the $2 n$ real-valued column vector obtained by concatenating the real and imaginary part of $\mathbf{s}_{v}$ i.e.

$$
\tilde{\mathbf{s}}_{v} \triangleq\left[\begin{array}{c}
\Re e\left(s_{1}^{(v)}\right) \\
\vdots \\
\Re e\left(s_{n}^{(v)}\right) \\
\Im m\left(s_{1}^{(v)}\right) \\
\vdots \\
\Im m\left(s_{n}^{(v)}\right)
\end{array}\right] .
$$

Let us also introduce the vectorization operator, $\operatorname{vec}\{$.$\} , ob-$ tained by stacking all columns of a matrix on top of each other. Using the property of the vec $\{$.$\} operator in equations$ (10) and (3) [37], it can be shown that

$$
\tilde{\mathbf{x}}_{v}=\underline{\mathbf{W G}} \tilde{\mathbf{s}}_{v}+\tilde{\mathbf{n}}_{v}
$$

where the $2 n_{t} l$-dimensional column vectors $\tilde{\mathbf{x}}_{v}$ and $\tilde{\mathbf{n}}_{v}$, the $2 n_{t} l \times 2 n_{t} l$ matrix $\underline{\mathbf{W}}$ and the $2 n_{t} l \times 2 n$ matrix $\mathbf{G}$ are respectively defined by

$$
\begin{aligned}
& \tilde{\mathbf{x}}_{v} \triangleq\left[\begin{array}{c}
\Re e\left(\operatorname{vec}\left\{\mathbf{X}_{v}^{\mathrm{H}}\right\}\right) \\
\Im m\left(\operatorname{vec}\left\{\mathbf{X}_{v}^{\mathrm{H}}\right\}\right)
\end{array}\right] \\
& \tilde{\mathbf{n}}_{v} \triangleq\left[\begin{array}{c}
\Re e\left(\operatorname{vec}\left\{\mathbf{N}_{v}^{\mathrm{H}}\right\}\right) \\
\Im m\left(\operatorname{vec}\left\{\mathbf{N}_{v}^{\mathrm{H}}\right\}\right)
\end{array}\right] \\
& \mathbf{G} \triangleq\left[\begin{array}{ccc}
\Re e\left(\operatorname{vec}\left\{\mathbf{A}_{1}^{\mathrm{H}}\right\}\right) & \cdots & \Re e\left(\operatorname{vec}\left\{\mathbf{A}_{2 n}^{\mathrm{H}}\right\}\right) \\
\Im m\left(\operatorname{vec}\left\{\mathbf{A}_{1}^{\mathrm{H}}\right\}\right) & \cdots & \Im m\left(\operatorname{vec}\left\{\mathbf{A}_{2 n}^{\mathrm{H}}\right\}\right)
\end{array}\right] \\
& \underline{\mathbf{W}} \triangleq\left[\begin{array}{cc}
\Re e\left(\mathbf{W}^{\mathrm{T}}\right) \otimes \mathbf{I}_{l} & -\Im m\left(\mathbf{W}^{\mathrm{T}}\right) \otimes \mathbf{I}_{l} \\
\Im m\left(\mathbf{W}^{\mathrm{T}}\right) \otimes \mathbf{I}_{l} & \Re e\left(\mathbf{W}^{\mathrm{T}}\right) \otimes \mathbf{I}_{l}
\end{array}\right] .
\end{aligned}
$$

As $\mathbf{W}$ is a unitary matrix, it is demonstrated in appendix A that $\underline{\mathbf{W}}$ is orthogonal i.e. $\underline{\mathbf{W}}^{\mathrm{T}} \underline{\mathbf{W}}=\mathbf{I}_{2 n_{t} l}$. If the unitary matrix $\mathbf{W}$ is known at the receiver side, the transmitted symbols can be recovered with a linear Zero-Forcing (ZF) equalizer. The
ZF equalizer computes an inverse matrix to compensate the combined effects of the channel and space-time coding i.e.

$$
\left[\begin{array}{c}
\Re e\left(\widehat{s}_{1}^{(v)}\right) \\
\vdots \\
\Re e\left(\widehat{s}_{n}^{(v)}\right) \\
\Im m\left(\widehat{s}_{1}^{(v)}\right) \\
\vdots \\
\Im m\left(\widehat{s}_{n}^{(v)}\right)
\end{array}\right]=\mathbf{G}^{\dagger} \underbrace{\left[\begin{array}{cc}
\Re e(\mathbf{W}) \otimes \mathbf{I}_{l} & \Im m(\mathbf{W}) \otimes \mathbf{I}_{l} \\
-\Im m(\mathbf{W}) \otimes \mathbf{I}_{l} & \Re e(\mathbf{W}) \otimes \mathbf{I}_{l}
\end{array}\right]}_{\underline{\mathbf{W}}^{\mathrm{T}}} \tilde{\mathbf{x}}_{v}
$$

where the $2 n \times 2 n_{t} l$ matrix $\mathbf{G}^{\dagger}$ denotes the pseudo-inverse of $\mathbf{G}\left(\mathbf{G}^{\dagger} \mathbf{G}=\mathbf{I}_{2 n}\right)$ and $\widehat{s}_{k}^{(v)}$ is the $k^{t h}$ estimated symbol of the $v^{\text {th }}$ block. Using (17), $\widehat{s}_{k}^{(v)}$ can be expressed as

$$
\widehat{s}_{k}^{(v)}=\left[\mathbf{e}_{k}^{(n)} j \mathbf{e}_{k}^{(n)}\right] \mathbf{G}^{\dagger} \underline{\mathbf{W}}^{\mathrm{T}} \tilde{\mathbf{x}}_{v} .
$$

In a blind context, the unitary matrix $\mathbf{W}$ is unknown at the receiver side. To estimate $\mathbf{W}$, this study exploits the statistical independence of the equalized symbols. More precisely, the unitary matrix $\mathbf{W}$ is estimated by maximizing the statistical independence of the Zero-Forcing equalized symbols, $\widehat{s}_{k}^{(v)}$.

2) Kurtosis-based cost function: A simple approach to maximize the statistical independence of $\widehat{s}_{k}^{(v)}$ is to maximize the nongaussianity of $\widehat{s}_{k}^{(v)}$ [23]. One measure of nongaussianity of a random variable $s$ is the (unnormalized) Kurtosis, $\mathcal{K}[s]$, which is defined as

$$
\mathcal{K}[s] \triangleq \mathcal{E}\left[|s|^{4}\right]-2\left(\mathcal{E}\left[|s|^{2}\right]\right)^{2}-\mathcal{E}[s s] \mathcal{E}\left[s^{*} s^{*}\right] .
$$

It follows that the unitary matrix $\mathbf{W}$ can be estimated by maximizing the function $\sum_{k=1}^{n}\left|\mathcal{K}\left[\widehat{s}_{k}^{(v)}\right]\right|$ where $|$.$| denotes the$ absolute value. It should be noted that in the most practical cases, the sign of the kurtosis is assumed to be known and the same for all the transmitted symbols. In particular, it is shown in reference [38] that the cumulant of most of the digital modulation (ASK, PSK and QAM) are negative. Therefore, an estimate of $\mathbf{W}$, denoted $\widehat{\mathbf{W}}$, can be obtained as follows

$$
\widehat{\mathbf{W}}:\left\{\begin{array}{rl}
\min _{\mathbf{W}} & \mathcal{J}(\mathbf{W})=\sum_{k=1}^{n} \mathcal{K}\left[\widehat{s}_{k}^{(v)}\right] \\
\text { subject to } \quad \mathbf{W W}^{\mathrm{H}}=\mathbf{I}_{n_{t}}
\end{array}\right.
$$

where $\mathcal{J}(\mathbf{W})$ is a real-valued cost function which depends on the $n_{t} \times n_{t}$ complex-valued matrix $\mathbf{W}$. It should be noted that criterion (20) has already appeared in literature for simpler channel estimation problems. In particular, it has been employed for classical ICA problems, where $\mathcal{C}\left(\mathbf{s}_{v}\right)=\mathbf{s}_{v}\left[{ }^{[39]-}\right.$ [42]. In our study, an extension to STBC systems is obtained by applying criterion (20) on the Zero-Forcing space-time equalized symbols $\widehat{s}_{k}^{(v)}$ in (18).

\section{Algorithm Implementation}

In this section, the focus is on the minimization of the realvalued cost function $\mathcal{J}: \mathbb{C}^{n_{t} \times n_{t}} \rightarrow \mathbb{R}$ under the unitary constraint $\mathbf{W} \mathbf{W}^{\mathrm{H}}=\mathbf{I}_{n_{t}}$. As no closed form solution exists, a Steepest-Descent (SD) approach is employed. To perform a descent step, SD algorithm requires the computation of the gradient. The gradient expression has been provided in several studies for classical ICA problems [42], [43], however, 
its expression is no longer valid for STBC systems. In the subsection IV.IV-A, the gradient expression is established for STBC systems. Then, two constrained-minimization SD algorithms are described in subsection IV.IV-B.

\section{A. Expression of the gradient in the Euclidean space}

In the Euclidean space, the gradient of the cost function $\mathcal{J}(\mathbf{W})$ is the $n_{t} \times n_{t}$ matrix $\Gamma_{\mathbf{W}}$ which is defined as [44]

$$
\Gamma_{\mathbf{W}}=\frac{d \mathcal{J}(\mathbf{W})}{d \mathbf{W}^{*}}
$$

where:

$$
\frac{d \mathcal{J}(\mathbf{W})}{d \mathbf{W}^{*}} \triangleq \frac{1}{2}\left(\frac{d \mathcal{J}(\mathbf{W})}{d \Re e(\mathbf{W})}+j \frac{d \mathcal{J}(\mathbf{W})}{d \Im m(\mathbf{W})}\right) .
$$

Let us denote, $w_{p l}$, the element on the $p^{\text {th }}$ row and the $l^{\text {th }}$ column of matrix $\mathbf{W}$. Using the $n_{t} \times n_{t}$ elementary matrix $\mathbf{E}_{p l}^{\left(n_{t}\right)}, \Gamma_{\mathbf{W}}$ can be expressed as

$$
\Gamma_{\mathbf{W}}=\sum_{p=1}^{n_{t}} \sum_{l=1}^{n_{t}} \frac{1}{2} \mathbf{E}_{p l}^{\left(n_{t}\right)}\left(\frac{d \mathcal{J}(\mathbf{W})}{d \Re e\left(w_{p l}\right)}+j \frac{d \mathcal{J}(\mathbf{W})}{d \Im m\left(w_{p l}\right)}\right) \text { (23) }
$$

From (20), it follows that

$$
\Gamma_{\mathbf{W}}=\sum_{p=1}^{n_{t}} \sum_{l=1}^{n_{t}} \sum_{k=1}^{n} \frac{1}{2} \mathbf{E}_{p l}^{\left(n_{t}\right)}\left(\frac{d \mathcal{K}\left[\widehat{s}_{k}^{(v)}\right]}{d \Re e\left(w_{p l}\right)}+j \frac{d \mathcal{K}\left[\widehat{s}_{k}^{(v)}\right]}{d \Im m\left(w_{p l}\right)}\right)(24)
$$

where $\widehat{s}_{k}$ is given by (18). By interchanging the order of derivative and expectation [45], the derivative of the (unnormalized) Kurtosis with respect to a complex element $x$ is given by

$$
\begin{aligned}
\frac{d \mathcal{K}\left[\widehat{s}_{k}\right]}{d x} & =2 \mathcal{E}\left[\left|\widehat{s}_{k}\right|^{2}\left(\widehat{s}_{k} \frac{d \widehat{s}_{k}^{*}}{d x}+\widehat{s}_{k}^{*} \frac{d \widehat{s}_{k}}{d x}\right)\right] \\
& -4 \mathcal{E}\left[\left|\widehat{s}_{k}\right|^{2}\right] \mathcal{E}\left[\widehat{s}_{k} \frac{d \widehat{s}_{k}^{*}}{d x}+\widehat{s}_{k}^{*} \frac{d \widehat{s}_{k}}{d x}\right] \\
& -2\left(\mathcal{E}\left[\widehat{s}_{k}^{2}\right] \mathcal{E}\left[\widehat{s}_{k}^{*} \frac{d \widehat{s}_{k}^{*}}{d x}\right]+\mathcal{E}\left[\widehat{s}_{k}^{2 *}\right] \mathcal{E}\left[\widehat{s}_{k} \frac{d \widehat{s}_{k}}{d x}\right]\right)(25)
\end{aligned}
$$

From (18), the $n_{t} \times n_{t}$ matrix $\Gamma_{\mathbf{W}}$ can be expressed as

$$
\begin{aligned}
\Gamma_{\mathbf{W}} & =\sum_{p=1}^{n_{t}} \sum_{l=1}^{n_{t}} \sum_{k=1}^{n} \mathbf{E}_{p l}^{\left(n_{t}\right)}\left(\mathcal{E}\left[\left|\widehat{s}_{k}^{(v)}\right|^{2}\left(\widehat{s}_{k}^{(v)} \mathbf{q}_{p l k}^{(2)}+\widehat{s}_{k}^{(v)} \mathbf{q}_{p l k}^{(1)}\right) \tilde{\mathbf{x}}_{v}\right]\right. \\
& -2 \mathcal{E}\left[\left|\widehat{s}_{k}^{(v)}\right|^{2}\right] \mathcal{E}\left[\left(\widehat{s}_{k}^{(v)} \mathbf{q}_{p l k}^{(2)}+\widehat{s}_{k}^{*(v)} \mathbf{q}_{p l k}^{(1)}\right) \tilde{\mathbf{x}}_{v}\right] \\
& \left.-\mathcal{E}\left[\widehat{s}_{k}^{(v)}\right] \mathcal{E}\left[\widehat{s}_{k}^{(v)} \mathbf{q}_{p l k}^{(2)} \tilde{\mathbf{x}}_{v}\right]-\mathcal{E}\left[\widehat{s}_{k}^{2 *(v)}\right] \mathcal{E}\left[\widehat{s}_{k}^{(v)} \mathbf{q}_{p l k}^{(1)} \tilde{\mathbf{x}}_{v}\right]\right)
\end{aligned}
$$

where the $2 n_{t} l$-dimensional column vector $\tilde{\mathbf{x}}_{v}$ is defined in (13) and where the $2 n_{t} l$-dimensional row vectors $\mathbf{q}_{p l k}^{(1)}$ and $\mathbf{q}_{\text {plk }}^{(2)}$ are given respectively by

$$
\begin{aligned}
& \mathbf{q}_{p l k}^{(1)}=\left[\mathbf{e}_{k}^{(n)} j \mathbf{e}_{k}^{(n)}\right] \mathbf{G}^{\dagger}\left[\begin{array}{cc}
\mathbf{E}_{p l}^{\left(n_{t}\right)} \otimes \mathbf{I}_{l} & j \mathbf{E}_{p l}^{\left(n_{t}\right)} \otimes \mathbf{I}_{l} \\
-j \mathbf{E}_{p l}^{\left(n_{t}\right)} \otimes \mathbf{I}_{l} & \mathbf{E}_{p l}^{\left(n_{t}\right)} \otimes \mathbf{I}_{l}
\end{array}\right] \\
& \mathbf{q}_{p l k}^{(2)}=\left[\mathbf{e}_{k}^{(n)}-j \mathbf{e}_{k}^{(n)}\right] \mathbf{G}^{\dagger}\left[\begin{array}{cc}
\mathbf{E}_{p l}^{\left(n_{t}\right)} \otimes \mathbf{I}_{l} & j \mathbf{E}_{p l}^{\left(n_{t}\right)} \otimes \mathbf{I}_{l} \\
-j \mathbf{E}_{p l}^{\left(n_{t}\right)} \otimes \mathbf{I}_{l} & \mathbf{E}_{p l}^{\left(n_{t}\right)} \otimes \mathbf{I}_{l}
\end{array}\right](28)
\end{aligned}
$$

Remark 1: In practice, the signals are assumed to be ergodic; that means that the expectation operator $\mathcal{E}[\cdot]$ in (26) can be approximated by a time-average.

\section{B. Constrained minimization algorithm}

Several SD algorithms for the minimization of a real-valued cost function under the unitary constraint have been proposed in literature. In this subsection, two algorithms are described.

For constrained-minimization, classical approaches solve the optimization problem on the Euclidean space by using gradient-based algorithms [23], [42], [46]. At each iteration step, an update of $\mathbf{W}$ is performed in the direction of the negative gradient. Then, a symmetric orthogonalization is applied to restore the unitary constraint of $\mathbf{W}$. This two-step approach is described in the algorithm 1 for a fixed step size ${ }^{2} \mu$. The major drawback of the Euclidean SD is that it can lead to undesired suboptimal solutions [47], [48].

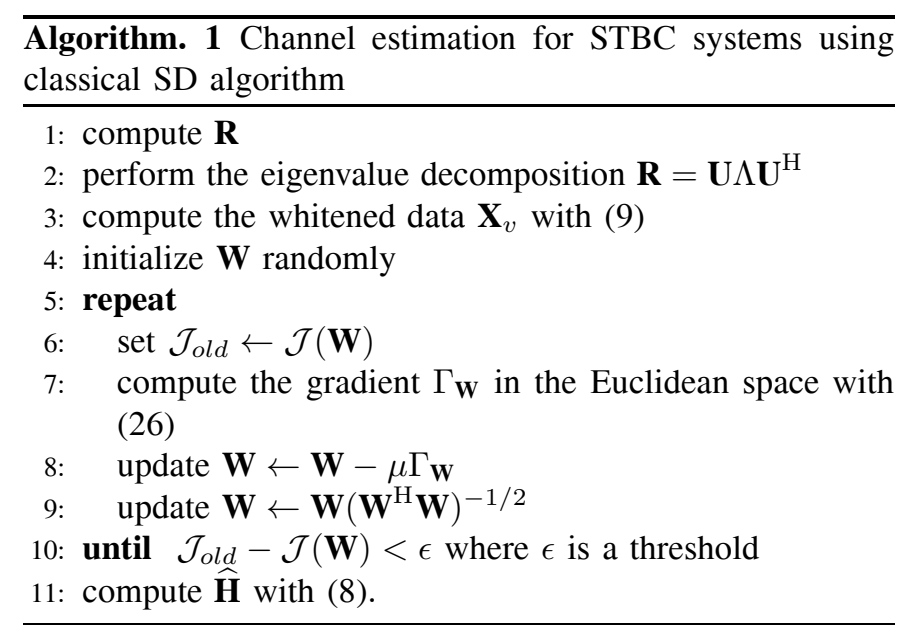

Recently, major improvements have been obtained by taking into account the geometrical aspect of the optimization problem. Nongeodesic and geodesic approaches have been proposed in [48], [49]. Coupled with Armijo step size rule [50], these algorithms always converge to a local minimum if it is not initialized at a stationary point. In the following equations, the geodesic SD algorithm [48] is chosen since it has lower computational complexity than the nongeodesic one. The geodesic SD algorithm moves towards the SD gradient direction, $\nabla_{\mathbf{W}}$, in the Riemannian space. This direction can be expressed as [48]

$$
\nabla_{\mathbf{W}}=\Gamma_{\mathbf{W}} \mathbf{W}^{\mathrm{H}}-\mathbf{W} \Gamma_{\mathbf{W}}^{\mathrm{H}}
$$

where $\Gamma_{\mathbf{W}}$ is the gradient in the Euclidean space (see (26)). Then, the update rule is given by

$$
\mathbf{W} \leftarrow \exp \left(\mu \nabla_{\mathbf{W}}\right) \mathbf{W}
$$

where $\exp (\cdot)=\sum_{k=0}^{\infty}(\cdot)^{k} / k !$ is the matrix exponential and $\mu$ corresponds to the step size. Using the Armijo step size rule, the algorithm almost always converges to a local minimum. The geodesic SD algorithm with the Armijo step size rule is described in the algorithm 2.

Figures 1 and 2 illustrate the convergence of algorithm 2 for a STBC system. The STBC system employs Alamouti coding

\footnotetext{
${ }^{2}$ As discussed in [47], line search optimization is not well-adapted for Euclidean SD with the projection method.
} 

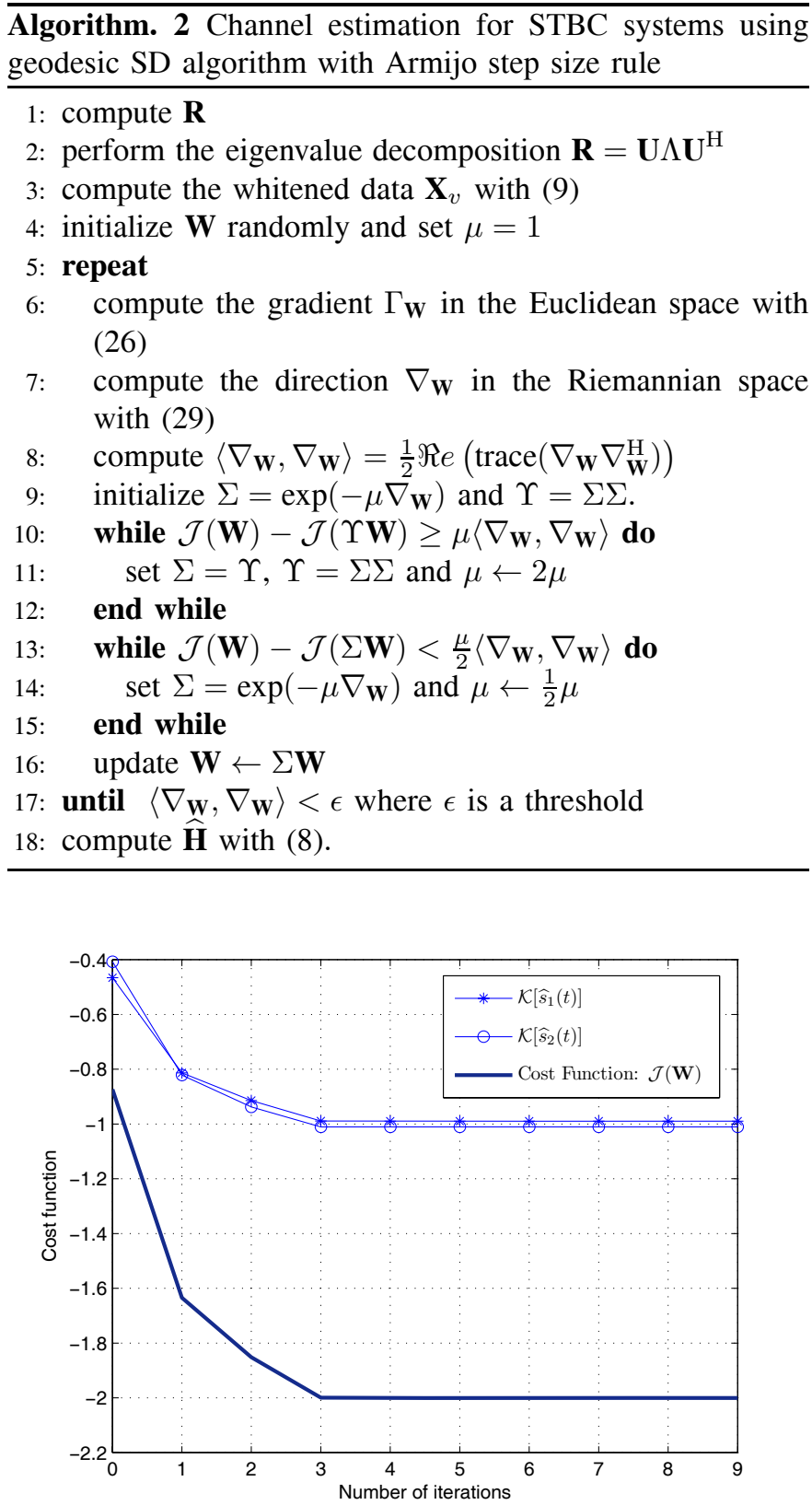

Fig. 1: Cost function $\mathcal{J}(\mathbf{W})$ versus iteration.

( $n_{t}=n=l=2$ ) and QPSK modulation. The number of transmitted blocks, the number of receiver antennae and the Signal to Noise Ratio (SNR) are equal to $N_{b}=512, n_{r}=4$ and $20 \mathrm{~dB}$, respectively, and the threshold is fixed at $\epsilon=10^{-5}$. Figure 1 displays $\mathcal{K}\left[\widehat{s}_{1}\right], \mathcal{K}\left[\widehat{s}_{2}\right]$ and $\mathcal{J}(\mathbf{W})$ with respect to the iteration number. The figure shows that the cost function is minimized after 9 iterations. The kurtosis $\mathcal{K}\left[\widehat{s}_{1}\right]$ and $\mathcal{K}\left[\widehat{s}_{2}\right]$ converge to -1 which is the kurtosis of QPSK modulation [38]. Figure 2 shows the constellation of the symbols $\widehat{s}_{1}$ and $\widehat{s}_{2}$ in the complex plane before and after convergence. After convergence, it should be noted that the constellation of the equalized symbols is phase-rotated as compared to the QPSK constellation. However, as opposed to the classical ICA model, the phase rotation ambiguities of $\widehat{s}_{1}$ and $\widehat{s}_{2}$ are not independent. The effect of the STBC structure on the channel ambiguities is studied in the following section.

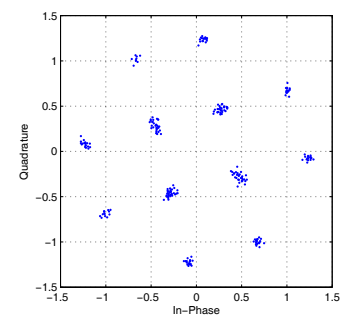

(a) After Whitening: $\widehat{s}_{1}$

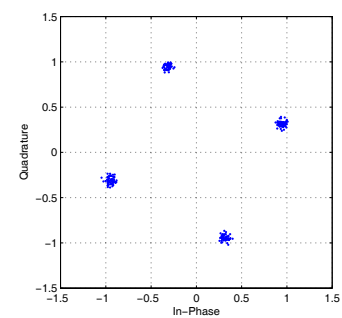

(c) Iteration 9: $\widehat{s}_{1}$

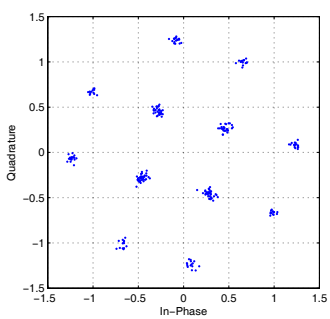

(b) After Whitening: $\widehat{s}_{2}$

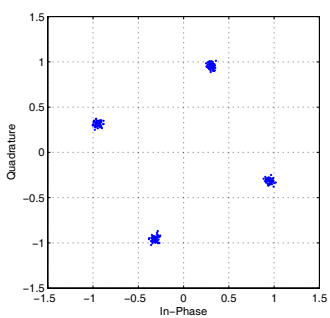

(d) Iteration 9: $\widehat{s}_{2}$
Fig. 2: Constellation of $\widehat{s}_{1}$ and $\widehat{s}_{2}$

\section{Remaining Ambiguities}

For the classical ICA model, it is well known that the channel can be estimated up to a permutation and phase rotation ambiguities [23], [51]. For STBC systems, the proposed method reduces the number of channel ambiguities by exploiting the spatio-temporal redundancy of the transmitted symbols in (18).

Theorem 1: Let us consider three matrices $\mathbf{M}, \mathbf{P}$ and $\mathbf{D}$ where $\mathbf{M}$ is an $n_{t} \times n_{t}$ unitary matrix, $\mathbf{P}$ is an $n \times n$ permutation matrix and $\mathbf{D}$ is an $n \times n$ diagonal matrix with entries of unit modulus $\left(\mathbf{D D}^{*}=\mathbf{I}_{n_{t}}\right)$. If these matrices satisfy

$$
\mathbf{M C}\left(\mathbf{s}_{v}\right)=\mathcal{C}\left(\mathbf{P D s}_{v}\right)
$$

for any $\mathbf{s}_{v}$, then $\mathbf{H} \mathbf{M}^{\mathrm{H}}$ is also a solution of the blind channel estimation problem.

Proof: From (4) and (31), one gets:

$$
\begin{aligned}
\mathbf{Y}_{v} & =\mathbf{H C}\left(\mathbf{s}_{v}\right)+\mathbf{B}_{v} \\
& =\mathbf{H} \mathbf{M}^{\mathrm{H}} \mathbf{M C}\left(\mathbf{s}_{v}\right)+\mathbf{B}_{v} \\
& =\mathbf{H} \mathbf{M}^{\mathrm{H}} \mathcal{C}\left(\mathbf{P D s}_{v}\right)+\mathbf{B}_{v} .
\end{aligned}
$$

As the elements of $\mathbf{s}_{v}$ are i.i.d, the elements of the vector $\mathbf{P D s}_{v}$ are also i.i.d. Therefore $\mathbf{H M}^{\mathrm{H}}$ is a solution of the blind channel estimation problem.

Let us express the set $\Theta$, which contains all the matrices $\mathbf{M}$ satisfying (31), with respect to the coding matrices. Condition (31) can be described in a vector form as

$$
\underbrace{\left[\begin{array}{c}
\Re e\left(\operatorname{vec}\left\{\mathcal{C}^{\mathrm{H}}\left(\mathbf{s}_{v}\right) \mathbf{M}^{\mathrm{H}}\right\}\right) \\
\Im m\left(\operatorname{vec}\left\{\mathcal{C}^{\mathrm{H}}\left(\mathbf{s}_{v}\right) \mathbf{M}^{\mathrm{H}}\right\}\right)
\end{array}\right]}_{\mathbf{k}_{1}}=\underbrace{\left[\begin{array}{c}
\Re e\left(\operatorname{vec}\left\{\mathcal{C}^{\mathrm{H}}\left(\mathbf{P D} \mathbf{s}_{v}\right)\right\}\right) \\
\Im m\left(\operatorname{vec}\left\{\mathcal{C}^{\mathrm{H}}\left(\mathbf{P D} \mathbf{s}_{v}\right)\right\}\right)
\end{array}\right]}_{\mathbf{k}_{2}} .
$$

The $2 n_{t} l$-dimensional column vector $\operatorname{vec}\left\{\mathcal{C}^{\mathrm{H}}\left(\mathbf{s}_{v}\right) \mathbf{M}^{\mathrm{H}}\right\}$ can be expressed as

$$
\operatorname{vec}\left\{\mathcal{C}^{\mathrm{H}}\left(\mathbf{s}_{v}\right) \mathbf{M}^{\mathrm{H}}\right\}=\left(\mathbf{M}^{*} \otimes \mathbf{I}_{l}\right) \operatorname{vec}\left\{\mathcal{C}^{\mathrm{H}}\left(\mathbf{s}_{v}\right)\right\}
$$


TABLE I: Set of ambiguity matrices for different STBCs using $n_{t}=2,3,4$ transmit antennae. The matrices $\mathbf{M}_{1}-\mathbf{M}_{10}$ are defined in (40)-(49).

\begin{tabular}{|c||c|c|c|c|}
\hline $\begin{array}{c}\text { Design } \\
\text { approach }\end{array}$ & $\begin{array}{c}\text { Number of } \\
\text { transmit } \\
\text { antennae } \\
n_{t}\end{array}$ & $\begin{array}{c}\text { Number of } \\
\text { symbols } \\
\text { per block } \\
n\end{array}$ & $\begin{array}{c}\text { Code rate } \\
n / l\end{array}$ & $\begin{array}{c}\text { Set of ambiguity matrices } \\
\text { after channel estimation }\end{array}$ \\
\hline Spatial Multiplex. & $n_{t}$ & $n_{t}$ & $n_{t}$ & $\Theta=\{\mathbf{P D}\}$ \\
Alamouti [1] & 2 & 2 & 1 & $\Theta=\left\{\mathbf{M}_{1}(\theta), \mathbf{M}_{2}(\theta)\right\}$ \\
OSTBC [2] & 3 & 3 & $1 / 2$ & $\Theta=\left\{ \pm \mathbf{I}_{3}\right\}$ \\
OSTBC [32] & 3 & 3 & $3 / 4$ & $\Theta=\left\{ \pm \mathbf{I}_{3}\right\}$ \\
OSTBC [3] & 4 & 3 & $3 / 4$ & $\Theta=\left\{ \pm \mathbf{I}_{4}\right\}$ \\
OSTBC [2] & 4 & 4 & $1 / 2$ & $\Theta=\left\{ \pm \mathbf{I}_{4}, \pm \mathbf{M}_{4}(0), \pm \mathbf{M}_{5}(0), \pm \mathbf{M}_{6}(0)\right\}$ \\
QOSTBC [4] & 4 & 4 & 1 & $\Theta=\left\{\mathbf{M}_{3}(\theta), \mathbf{M}_{4}(\theta), \mathbf{M}_{5}(\theta), \mathbf{M}_{6}(\theta)\right\}$ \\
NOSTBC [52] & 4 & 4 & 1 & $\Theta=\left\{\mathbf{M}_{7}\left(\theta_{1}, \theta_{2}\right), \mathbf{M}_{8}\left(\theta_{1}, \theta_{2}\right), \mathbf{M}_{9}\left(\theta_{1}, \theta_{2}\right), \mathbf{M}_{10}\left(\theta_{1}, \theta_{2}\right)\right\}$ \\
\hline
\end{tabular}

As $\Re e\left(\mathbf{M}^{*}\right)=\Re e(\mathbf{M})$ and $\Im m\left(\mathbf{M}^{*}\right)=-\Im m(\mathbf{M}), \mathbf{k}_{1}$ can be written in a linear form as

$$
\mathbf{k}_{1}=\left[\begin{array}{cc}
\Re e(\mathbf{M}) \otimes \mathbf{I}_{l} & \Im m(\mathbf{M}) \otimes \mathbf{I}_{l} \\
-\Im m(\mathbf{M}) \otimes \mathbf{I}_{l} & \Re e(\mathbf{M}) \otimes \mathbf{I}_{l}
\end{array}\right] \mathbf{G} \tilde{\mathbf{s}}_{v} .
$$

The right term in (33) can also be expressed into a linear form as

$$
\begin{aligned}
\mathbf{k}_{2} & =\mathbf{G}\left[\begin{array}{c}
\Re e\left(\operatorname{vec}\left\{\mathbf{P D s}_{v}\right\}\right) \\
\Im m\left(\operatorname{vec}\left\{\mathbf{P D s}_{v}\right\}\right)
\end{array}\right] \\
& =\mathbf{G}\left[\begin{array}{cc}
\mathbf{P} \Re e(\mathbf{D}) & -\mathbf{P} \Im m(\mathbf{D}) \\
\mathbf{P} \Im m(\mathbf{D}) & \mathbf{P} \Re e(\mathbf{D})
\end{array}\right] \tilde{\mathbf{s}}_{v} .
\end{aligned}
$$

Using (35) and (36), (33) can be simplified as

$$
\underline{\mathbf{M}}^{\mathrm{T}} \mathbf{G} \tilde{\mathbf{s}}_{v}=\mathbf{G}\left[\begin{array}{cc}
\mathbf{P} \Re e(\mathbf{D}) & -\mathbf{P} \Im m(\mathbf{D}) \\
\mathbf{P} \Im m(\mathbf{D}) & \mathbf{P} \Re e(\mathbf{D})
\end{array}\right] \tilde{\mathbf{s}}_{v}
$$

where $\underline{\mathbf{M}}^{\mathrm{T}}$ is an $2 n_{t} l \times 2 n_{t} l$ matrix with real elements, which is defined as

$$
\underline{\mathbf{M}}^{\mathrm{T}}=\left[\begin{array}{cc}
\Re e(\mathbf{M}) \otimes \mathbf{I}_{l} & \Im m(\mathbf{M}) \otimes \mathbf{I}_{l} \\
-\Im m(\mathbf{M}) \otimes \mathbf{I}_{l} & \Re e(\mathbf{M}) \otimes \mathbf{I}_{l}
\end{array}\right] .
$$

As (37) must be satisfied for any $\mathbf{s}_{v}$, one obtains

$$
\mathbf{G}^{\dagger} \underline{\mathbf{M}}^{\mathrm{T}} \mathbf{G}=\left[\begin{array}{cc}
\mathbf{P} \Re e(\mathbf{D}) & -\mathbf{P} \Im m(\mathbf{D}) \\
\mathbf{P} \Im m(\mathbf{D}) & \mathbf{P} \Re e(\mathbf{D})
\end{array}\right]
$$

Finally, the following result is obtained

Theorem 2: For any STBC $\mathcal{C}$, the set $\Theta$ of ambiguity matrices is the one containing all the $n_{t} \times n_{t}$ matrices $\mathbf{M}$ satisfying (39) where $\mathbf{P}$ is a $n_{t} \times n_{t}$ permutation matrix and $\mathbf{D}$ is a $n_{t} \times n_{t}$ diagonal matrix with entries of unit modulus.

It should be noted that the condition (39) depends on the matrix $\mathbf{G}$ which only depends on the STBC (see (15)). Unfortunately, it appears to be difficult to find the exact relationship between $\mathbf{G}$ and the matrices $\mathbf{M}, \mathbf{P}$ and $\mathbf{D}$. To provide a clear relationship between these matrices, we have performed several Monte-Carlo simulations with the Rayleigh MIMO channel. Table I provides the set $\Theta$ of ambiguity matrices for several STBCs using $n_{t}=\{2,3,4\}$ transmit antennae. In Table I, matrices $\mathbf{M}_{1}-\mathbf{M}_{10}$ are equal to

$$
\begin{aligned}
\mathbf{M}_{1}(\theta) & =\left[\begin{array}{cc}
e^{j \theta} & 0 \\
0 & e^{-j \theta}
\end{array}\right] \\
\mathbf{M}_{2}(\theta) & =\left[\begin{array}{cc}
0 & e^{j \theta} \\
-e^{-j \theta} & 0
\end{array}\right] \\
\mathbf{M}_{3}(\theta) & =\left[\begin{array}{cc}
\mathbf{M}_{1}(\theta) & \mathbf{0}_{2} \\
\mathbf{0}_{2} & \mathbf{M}_{1}(-\theta)
\end{array}\right] \\
\mathbf{M}_{4}(\theta) & =\left[\begin{array}{cc}
\mathbf{M}_{2}(\theta) & \mathbf{0}_{2} \\
\mathbf{0}_{2} & \mathbf{M}_{2}(-\theta)
\end{array}\right] \\
\mathbf{M}_{5}(\theta) & =\left[\begin{array}{cc}
\mathbf{0}_{2} & \mathbf{M}_{1}(\theta) \\
-\mathbf{M}_{1}(-\theta) & \mathbf{0}_{2}
\end{array}\right] \\
\mathbf{M}_{6}(\theta) & =\left[\begin{array}{cc}
\mathbf{0}_{2} & \mathbf{M}_{2}(\theta) \\
-\mathbf{M}_{2}(-\theta) & \mathbf{0}_{2}
\end{array}\right] \\
\mathbf{M}_{7}\left(\theta_{1}, \theta_{2}\right) & =\left[\begin{array}{cc}
\mathbf{M}_{1}\left(\theta_{1}\right) & \mathbf{0}_{2} \\
\mathbf{0}_{2} & \mathbf{M}_{1}\left(\theta_{2}\right)
\end{array}\right] \\
\mathbf{M}_{8}\left(\theta_{1}, \theta_{2}\right) & =\left[\begin{array}{cc}
\mathbf{M}_{1}\left(\theta_{1}\right) & \mathbf{0}_{2} \\
\mathbf{0}_{2} & \mathbf{M}_{2}\left(\theta_{2}\right)
\end{array}\right] \\
\mathbf{M}_{9}\left(\theta_{1}, \theta_{2}\right) & =\left[\begin{array}{cc}
\mathbf{M}_{2}\left(\theta_{1}\right) & \mathbf{0}_{2} \\
\mathbf{0}_{2} & \mathbf{M}_{1}\left(\theta_{2}\right)
\end{array}\right] \\
\mathbf{M}_{10}\left(\theta_{1}, \theta_{2}\right) & =\left[\begin{array}{cc}
\mathbf{M}_{2}\left(\theta_{1}\right) & \mathbf{0}_{2} \\
\mathbf{0}_{2} & \mathbf{M}_{2}\left(\theta_{2}\right)
\end{array}\right] .
\end{aligned}
$$

Let us emphasize the differences between Table I and the tables reported in [17], [18], [21], [22]. Tables reported in [17], [18], [21], [22] focus on the blind channel-identifiability condition for subspace and SOS approaches. Without modification of the transmitter (precoding, pilot sequence), they show that subspace and SOS methods are unable to estimate the channel for $\geq 1$-rate STBCs and some specific low-rate STBCs. Unlike subspace and SOS approaches, the proposed method can be applied to the whole class of linear STBCs without any modification of the transmitter or the use of a pilot sequence. Moreover, unlike the general subspace methods [16], [17], it does not introduce additional ambiguities to those associated to the blind channel estimation problem. For example for the $\frac{3}{4}$-rate OSTBC using $n_{t}=3$ antennae, the proposed method can estimate the channel up to a sign whereas the subspace method introduces an unknown phase rotation $e^{j \theta}$ [16], [17].

\section{Simulation Results}

Monte-Carlo simulations were run to assess the performances of the algorithms 1 and 2 . Let us denote by $\mathbf{H}$ and $\widehat{\mathbf{H}}$ the original and estimated channel, respectively. After 
TABLE II: Spatial Multiplexing: Average Computation times for each algorithm.

\begin{tabular}{|l|c|c|c|}
\hline Algorithm SNR & $-10 \mathrm{~dB}$ & $0 \mathrm{~dB}$ & $10 \mathrm{~dB}$ \\
\hline Classical SD & $0.07 \mathrm{~s}$ & $0.11 \mathrm{~s}$ & $0.09 \mathrm{~s}$ \\
Geodesic SD & $0.34 \mathrm{~s}$ & $0.16 \mathrm{~s}$ & $0.09 \mathrm{~s}$ \\
JADE & $0.004 \mathrm{~s}$ & $0.004 \mathrm{~s}$ & $0.004 \mathrm{~s}$ \\
\hline
\end{tabular}

channel estimation, the remaining ambiguity is removed by post-multiplying $\widehat{\mathbf{H}}$ with $\widehat{\mathbf{M}}$ where

$$
\widehat{\mathbf{M}}=\arg \min _{\mathbf{M} \in \Theta}\|\mathbf{H}-\widehat{\mathbf{H}} \mathbf{M}\|_{F}^{2}
$$

and where the set of ambiguity matrices, $\Theta$, depends on the STBC (see Table I). After ambiguity removal, the estimated channel is denoted as $\widehat{\mathbf{H}}_{a}=\widehat{\mathbf{H}} \mathbf{M}$. Performances of the proposed blind algorithms were quantified through:

- the Normalized Mean Square Error (NMSE), which is defined as:

$$
N M S E=\frac{\left\|\mathbf{H}-\widehat{\mathbf{H}}_{a}\right\|_{F}^{2}}{\|\mathbf{H}\|_{F}^{2}} .
$$

- the average Symbol Error Rate (SER) obtained after ML decoding.

Each simulation was carried out under the following conditions: i) a Rayleigh distributed channel i.e. each element of $\mathbf{H}$ follows an i.i.d. circular Gaussian distribution with zeromean and unit-variance, ii) a QPSK modulation, iii) 512 transmitted blocks, iv) a temporally and spatially zero-mean white Gaussian additive noise with variance $\sigma^{2}$ (which is unknown at the receiver side), v) a threshold equal to $\epsilon=10^{-5}$ and vi) a receiver satisfying assumption AS1). Performances of the algorithms 1 and 2 were evaluated for several Signalto-Noise Ratios (SNRs) where the SNR was defined as [53]

$$
S N R=10 \log _{10}\left(n_{t} / \sigma^{2}\right) \text {. }
$$

For each SNR, two thousand Monte-Carlo simulations were performed to approximate the NMSE and SER. As there is no guarantee that the algorithms 1 and 2 will find the global minimum, performances of the proposed methods were also evaluated with multistart initialization. Multistart initialization procedure runs an algorithm several times with new random starting points and selects the estimated unitary matrix $\mathbf{W}$ which minimizes the cost-function $\mathcal{J}(\mathbf{W})$. In the following subsections, performances are presented for 3 different STBC systems.

\section{A. Spatial Multiplexing}

In this subsection, we consider the case of a Spatial Multiplexing system using $n_{t}=2$ transmit antennae. The transmitted blocks are given by

$$
\mathcal{C}(\mathbf{s})=\left[\begin{array}{l}
s_{1} \\
s_{2}
\end{array}\right] .
$$

For Spatial Multiplexing, the channel estimation problem reduces to the classical ICA problem. After channel estimation, the set of ambiguity matrices is given by $\Theta=\{\mathbf{P D}\}$ where

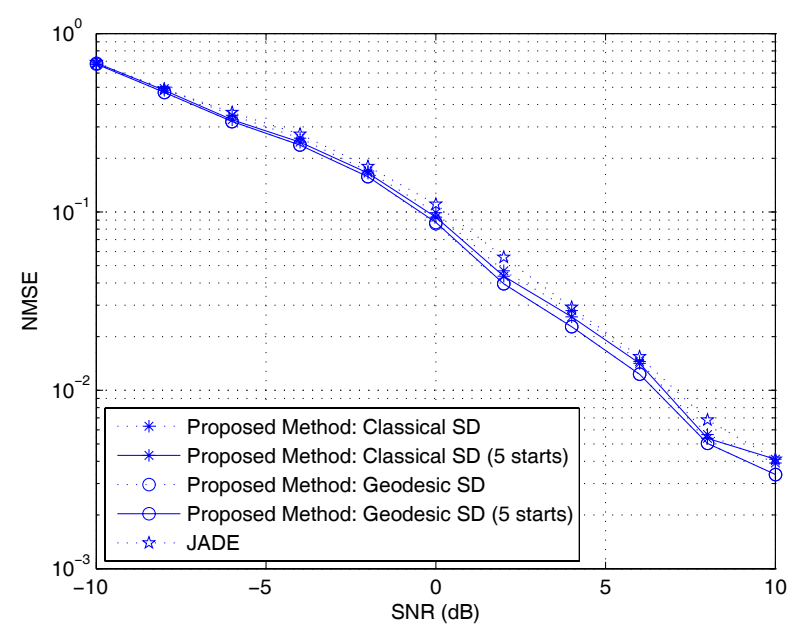

Fig. 3: Spatial Multiplexing: NMSE.

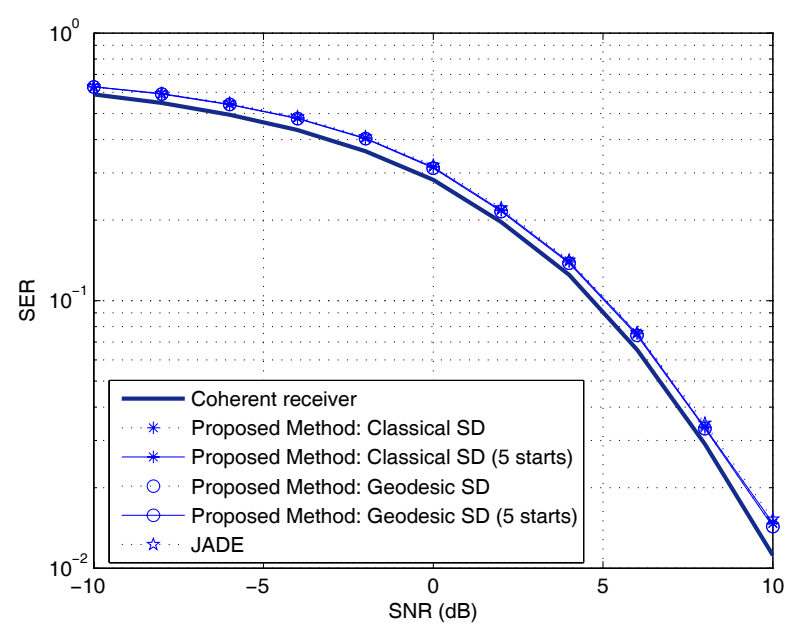

Fig. 4: Spatial Multiplexing: Symbol Error Rate.

$\mathbf{P}$ and $\mathbf{D}$ are permutation and phase matrices. Figures 3 and 4 present the performances of the algorithms 1 and 2 for a receiver composed of $n_{r}=3$ antennae. These two algorithms are compared with JADE [54]. Figure 3 displays the channel NMSE versus SNR. In this simulation, algorithms 1 and 2 always match or outperform the JADE algorithm, depending on the SNR. Figure 3 also indicates that the multistart initialization does not seem to improve the performances. Figure 4 presents the SER versus the SNR. The SER is compared to the one obtained with the coherent ML receiver (perfect CSI). It should be noted that the blind channel-estimation algorithms achieve near-optimal performances at high SNR since their SERs approach the ones of the coherent ML receiver. A comparison of the average computation times is shown in Table II for simulations implemented on a $2.6 \mathrm{GHz}$ Intel Pentium processor using Matlab. For multistart initialization approaches, the computation times must be multiplied by the number of random starts. Table II shows that the classical $\mathrm{SD}$ is less computationally demanding than the geodesic SD at low-SNR, but their computational complexities are similar at high-SNR. Table II also suggests that the JADE algorithm is significantly less complex than the proposed algorithms. 


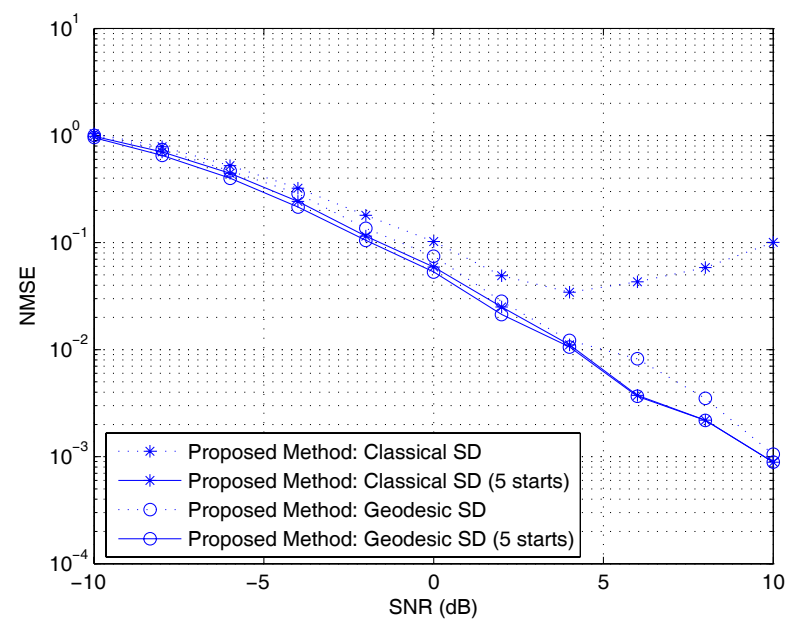

Fig. 5: Alamouti Coding: NMSE.

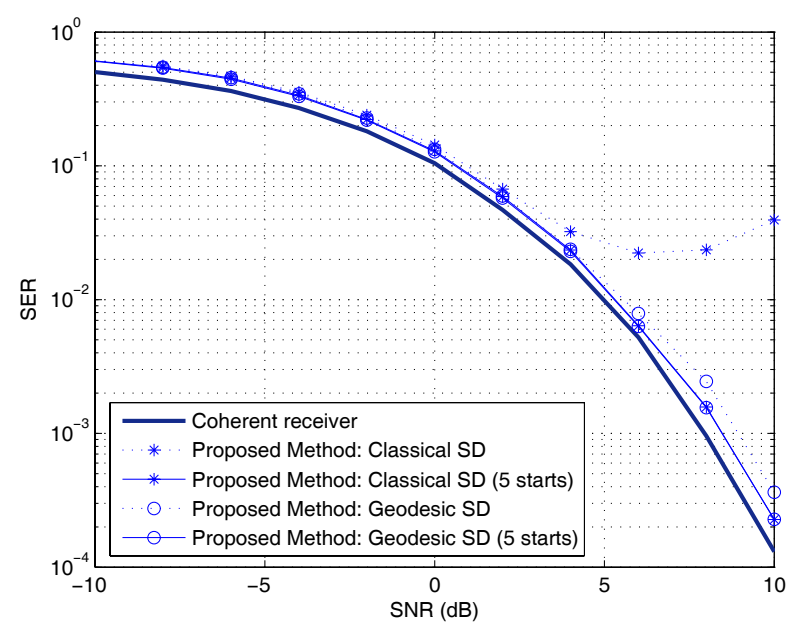

Fig. 6: Alamouti Coding: Symbol Error Rate.

TABLE III: Alamouti Coding: Average Computation times for each algorithm.

\begin{tabular}{|l|c|c|c|}
\hline Algorithm SNR & $-10 \mathrm{~dB}$ & $0 \mathrm{~dB}$ & $10 \mathrm{~dB}$ \\
\hline Classical SD & $0.10 \mathrm{~s}$ & $0.16 \mathrm{~s}$ & $0.12 \mathrm{~s}$ \\
Geodesic SD & $0.51 \mathrm{~s}$ & $0.21 \mathrm{~s}$ & $0.10 \mathrm{~s}$ \\
\hline
\end{tabular}

However, one should note that JADE is limited to SpatialMultiplexing systems and cannot be employed for more general settings.

\section{B. Alamouti Coding}

In this subsection, we consider a STBC system using the Alamouti Code. This Orthogonal code is defined by [1]

$$
\mathcal{C}(\mathbf{s})=\left[\begin{array}{cc}
s_{1} & -s_{2}^{*} \\
s_{2} & s_{1}^{*}
\end{array}\right]
$$

For Alamouti coding, the direct use of an ICA algorithm, like JADE, is irrelevant since the transmitted symbols between consecutive time instances are not independent. Furthermore, it is demonstrated in [16]-[18], [22] that subspace and SOS approaches cannot estimate the channel matrix when the transmitter employs Alamouti Coding. Regarding the proposed methods, Table I shows that the set of ambiguity matrices after channel estimation is $\Theta=\left\{\mathbf{M}_{1}(\theta), \mathbf{M}_{2}(\theta)\right\}$. Figure 5 displays the NMSE versus SNR for a receiver composed of $n_{r}=3$ antennae. Without multistart initialization, the geodesic SD clearly outperforms the classical SD since the latter exhibits an error floor at SNR greater than $4 \mathrm{~dB}$. This error floor is due to the fact that the Euclidean SD can lead to undesired suboptimal solutions even at high SNR [47], [48]. It should be observed that the multistart initialization strategy removes the error floor and improves the NMSE performances of the two proposed algorithms. Figure 6 compares the SER with the one obtained with a coherent ML receiver. As previously discussed, without multistart initialization, the performances of the Euclidean SD lead to an error floor at SNR greater than $4 \mathrm{~dB}$. However, it should be observed that algorithms 1 and 2 achieve near-optimal performance when a multistart initizalization is used. A comparison of the average computation times is shown in Table III. It should be noted that classical $\mathrm{SD}$ is less computationally demanding than the geodesic SD at low-SNR, but this trend is reversed at high SNR.

\section{C. $\frac{3}{4}$-rate OSTBC using 3 antennae}

In this subsection, we consider the case of a $\frac{3}{4}$-rate OSTBC using 3 antennae. This OSTBC is defined by [32]

$$
\mathcal{C}(\mathbf{s})=\left[\begin{array}{cccc}
s_{1} & 0 & s_{2} & -s_{3} \\
0 & s_{1} & s_{3}^{*} & s_{2}^{*} \\
-s_{2}^{*} & -s_{3} & s_{1} & 0
\end{array}\right]
$$

For this low-rate code, the channel can be estimated with subspace and SOS approaches. The remaining ambiguity reduces to a sign for the SOS approach [18] and to a rotation $e^{j \theta}$ for the subspace method [16], [17]. In the following figures, performances of the proposed algorithms are compared with the SOS-based method [18] for a receiver composed of $n_{r}=5$ antennae. Figure 7 displays the NMSE versus SNR. Without multiple-start initialization, it should be noted that the algorithms 1 and 2 exhibit an error floor at SNR greater than 6dB. For the Euclidean SD algorithm, the error floor is due to the fact that this approach can lead to undesired suboptimal solutions [47], [48]. For the Geodesic SD one, even if it always converges to a local minimum [47], the error floor is due to the fact that the local minimum does not necessarily coincide with the global one. As previously observed, multistart initialization removes the error floor and improves the performances of the two proposed algorithms. In particular, Figure 7 shows that for $S N R>0$, algorithms 1 and 2 with multistart initialization outperform the SOS method. Figure 8 shows that method [18] and the proposed multistart algorithms both achieve near-optimal SER performances for $S N R>0$. A comparison of the average computation times is presented in Table IV. It is shown that the SOS method is less computationally demanding than the proposed algorithms. Therefore, for SOS-identifiable OSTBCs, it seems that the closed-form SOS algorithm is definitely preferable since this algorithm does not suffer from convergence problems and it is less computationally expensive than the proposed approaches. However, it should be emphasized that the SOS method is 


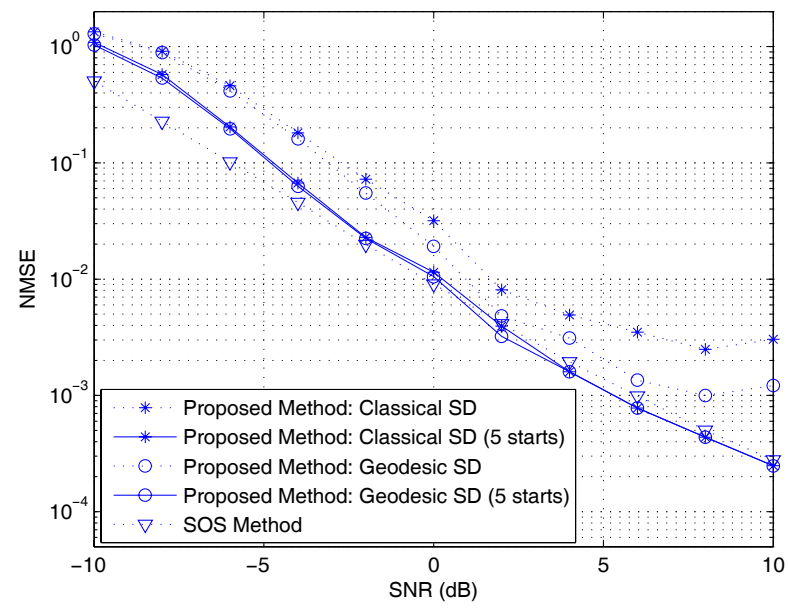

Fig. 7: 3/4-rate OSTBC: NMSE.

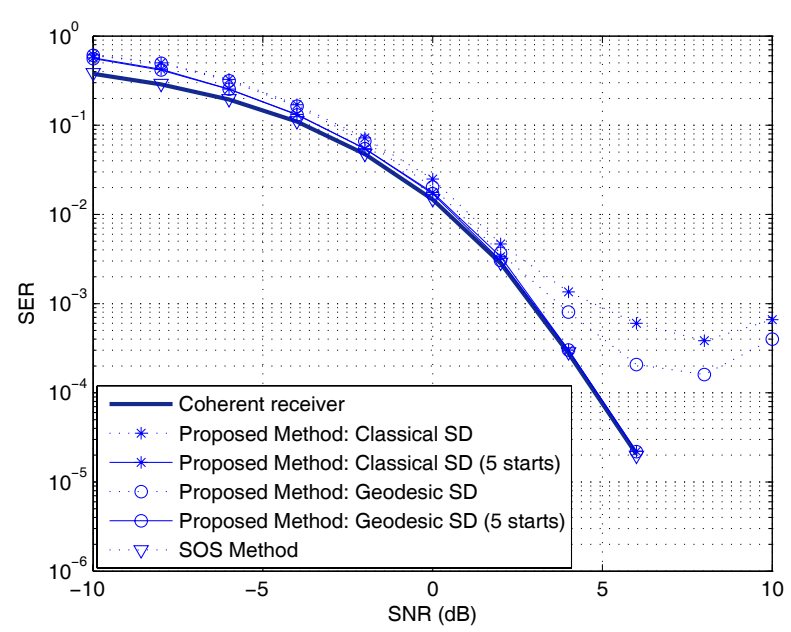

Fig. 8: 3/4-rate OSTBC: Symbol Error Rate.

TABLE IV: $\frac{3}{4}$-rate OSTBC: Average Computation times for each algorithm.

\begin{tabular}{|l|c|c|c|}
\hline Algorithm SNR & $-10 \mathrm{~dB}$ & $0 \mathrm{~dB}$ & $10 \mathrm{~dB}$ \\
\hline Classical SD & $1.32 \mathrm{~s}$ & $0.85 \mathrm{~s}$ & $0.78 \mathrm{~s}$ \\
Geodesic SD & $3.33 \mathrm{~s}$ & $0.62 \mathrm{~s}$ & $0.51 \mathrm{~s}$ \\
SOS method & $0.48 \mathrm{~s}$ & $0.48 \mathrm{~s}$ & $0.48 \mathrm{~s}$ \\
\hline
\end{tabular}

limited to a subclass of OSTBCs [22], whereas the proposed algorithms can be applied to the whole class of linear STBCs.

\section{CONCLUSION}

This paper proposed an original blind channel estimation algorithm for space-time block coding communications. The method is based on the minimization of a kurtosis-based cost function after Zero-Forcing equalization. The proposed method can be applied to the whole class of linear STBCs, whatever the code-rate and the modulation. This paper also presented the set of the remaining channel ambiguities for several STBCs using 2, 3 or 4 transmit antennae. The good performances of the proposed algorithm were demonstrated through computer simulations for different STBCs. In particular, simulations have shown that the proposed method matches or outperforms the JADE algorithm [54] for Spatial Multiplexing and matches the performances of the closed-form SOS approach [18] for identifiable OSTBCs.

\section{APPENDIX}

Let us consider the $2 n_{t} l \times 2 n_{t} l$ matrix $\underline{\mathbf{W}}$ defined in (12). As $(\mathbf{A} \otimes \mathbf{B})^{\mathrm{T}}=\mathbf{A}^{\mathrm{T}} \otimes \mathbf{B}^{\mathrm{T}}$, one gets:

$$
\begin{aligned}
\underline{\mathbf{W}}^{\mathrm{T}} \underline{\mathbf{W}} & =\left[\begin{array}{cc}
\Re e(\mathbf{W}) \otimes \mathbf{I}_{l} & \Im m(\mathbf{W}) \otimes \mathbf{I}_{l} \\
-\Im m(\mathbf{W}) \otimes \mathbf{I}_{l} & \Re e(\mathbf{W}) \otimes \mathbf{I}_{l}
\end{array}\right] \\
\times & {\left[\begin{array}{cc}
\Re e\left(\mathbf{W}^{\mathrm{T}}\right) \otimes \mathbf{I}_{l} & -\Im m\left(\mathbf{W}^{\mathrm{T}}\right) \otimes \mathbf{I}_{l} \\
\Im m\left(\mathbf{W}^{\mathrm{T}}\right) \otimes \mathbf{I}_{l} & \Re e\left(\mathbf{W}^{\mathrm{T}}\right) \otimes \mathbf{I}_{l}
\end{array}\right] }
\end{aligned}
$$

From the mixed product rule, it follows that:

$$
\underline{\mathbf{W}}^{\mathrm{T}} \underline{\mathbf{W}}=\left[\begin{array}{cc}
\mathbf{B}_{1} \otimes \mathbf{I}_{l} & -\mathbf{B}_{2} \otimes \mathbf{I}_{l} \\
\mathbf{B}_{2} \otimes \mathbf{I}_{l} & \mathbf{B}_{1} \otimes \mathbf{I}_{l}
\end{array}\right]
$$

where the $n_{t} \times n_{t}$ matrices $\mathbf{B}_{1}$ and $\mathbf{B}_{2}$ are given by:

$$
\begin{aligned}
& \mathbf{B}_{1}=\Re e(\mathbf{W}) \Re e\left(\mathbf{W}^{\mathrm{T}}\right)+\Im m(\mathbf{W}) \Im m\left(\mathbf{W}^{\mathrm{T}}\right) \\
& \mathbf{B}_{2}=\Re e(\mathbf{W}) \Im m\left(\mathbf{W}^{\mathrm{T}}\right)-\Im m(\mathbf{W}) \Re e\left(\mathbf{W}^{\mathrm{T}}\right)
\end{aligned}
$$

As $\mathbf{W}$ is a $n_{t} \times n_{t}$ unitary matrix, it satisfies $\mathbf{W} \mathbf{W}^{\mathrm{H}}=\mathbf{I}_{n_{t}}$. By expanding the real and imaginary parts, one gets:

$$
\begin{aligned}
\mathbf{W W}^{\mathrm{H}} & =(\Re e(\mathbf{W})+j \Im m(\mathbf{W})) \cdot\left(\Re e\left(\mathbf{W}^{\mathrm{T}}\right)-j \Im m\left(\mathbf{W}^{\mathrm{T}}\right)\right) \\
& =\mathbf{B}_{1}-j \mathbf{B}_{2}=\mathbf{I}_{n_{t}}
\end{aligned}
$$

By identification, it follows that $\mathbf{B}_{1}=\mathbf{I}_{n_{t}}$ and $\mathbf{B}_{2}=\mathbf{0}_{n_{t}}$. Finally (57) can be simplified as:

$$
\underline{\mathbf{W}}^{\mathrm{T}} \underline{\mathbf{W}}=\mathbf{I}_{2 n_{t} l}
$$

\section{REFERENCES}

[1] S. Alamouti, "A simple transmit diversity technique for wireless communication," IEEE J. Sel. Areas Commun., vol. 16, no. 8, pp. 1451-1458, 1998.

[2] V. Tarokh, H. Jafarkhani, and A. Calderbank, "Space time block codes from orthogonal designs," IEEE Trans. Inf. Theory, vol. 45, no. 5, pp. 744-765, 1999.

[3] G. Ganesan and P. Stoica, "Space-time block codes: a maximum SNR approach," IEEE Trans. Inf. Theory, vol. 47, no. 4, pp. 1650-1656, 2001.

[4] H. Jafarkhani, "A quasi-orthogonal space-time block code," IEEE Trans. Commun., vol. 49, no. 1, pp. 1-4, 2001.

[5] A. Boarui and D. Ionescu, "A class of nonorthogonal rate-one spacetime block codes with controlled interference," IEEE Trans. Wireless Commun., vol. 2, no. 2, pp. 270-276, 2003.

[6] H. Jafarkhani, Space-Time Coding: Theory and Practice. Cambridge University Press, 2005.

[7] G. Ganesan and P. Stoica, "Differential modulation using space-time block codes," IEEE Signal Process. Lett., vol. 9, no. 2, pp. 57-60, 2002.

[8] B. Hochwald and W. Sweldens, "Differential unitary space-time modulation," IEEE Trans. Commun., vol. 48, no. 12, pp. 2041-2052, 2000.

[9] B. Hughes, "Differential space-time modulation," IEEE Trans. Inf. Theory, vol. 46, no. 7, pp. 2567-2578, 2000.

[10] Y. Zhu and H. Jafarkhani, "Differential modulation based on quasiorthogonal codes," IEEE Trans. Wireless Commun., vol. 4, no. 6, pp. 3005-3017, 2005.

[11] E. Larsson, P. Stoica, and J. Li, "On maximum-likelihood detection and decoding for space-time coding systems," IEEE Trans. Signal Process., vol. 50, no. 4, pp. 937-944, 2002.

[12] — - "Orthogonal space-time block codes: maximum likelihood detection for unknown channels and unstructured intereferences," IEEE Trans. Signal Process., vol. 51, no. 2, pp. 362-372, 2003.

[13] W. Ma, B. Vo, T. Davidson, and P. Ching, "Blind ML detection of orthogonal space-time block codes: efficient high-performance implementations," IEEE Trans. Signal Process., vol. 54, no. 2, pp. 738-751, 2006. 
[14] Y. Li, C. Georghiades, and G. Huang, "Iterative maximum likelihood sequence estimation for space-time coded systems," IEEE Trans. Commun., vol. 49, no. 6, pp. 948-951, 2001.

[15] A. Gallo, E. Chiavaccini, F. Muratori, and G. Vitetta, "BEM-based SISO detection of orthogonal space-time block codes over frequency flatfading channels," IEEE Trans. Wireless Commun., vol. 3, no. 6, pp. 1885-1889, 2004.

[16] A. Swindlehurst and G. Leus, "Blind and semi-blind equalization for generalized space-time block codes," IEEE Trans. Signal Process., vol. 50, no. 10, pp. 2489-2498, 2002.

[17] N. Ammar and Z. Ding, "Blind channel identifiability for generic linear space-time block codes," IEEE Trans. Signal Process., vol. 55, no. 1, pp. 202-217, 2007.

[18] S. Shahbazpanahi, A. Gershman, and J. Manton, "Closed form blind MIMO channel estimation for othogonal space-time codes," IEEE Trans. Signal Process., vol. 53, no. 12, pp. 4506-4517, 2005.

[19] J. Via, I. Santamaria, A. Sezdin, and A. Paulraj, "SOS-based blind channel estimation algorithm under space-time block coded transmissions," in Eighth IEEE Workshop Signal Process. Advances Wireless Commun., Helsinki, Finland, June 2007.

[20] J. Via and I. Santamaria, "Correlation matching approaches for blind OSTBC channel estimation," IEEE Trans. Signal Process., vol. 56, no. 12, pp. 5950-5961, 2008.

[21] J. Via, I. Santamaria, and J. Perez, "Code combination for blind channel estimation in general MIMO-STBC systems," EURASIP J. Advances Signal Process., 2009.

[22] J. Via and I. Santamaria, "On the blind identifiablity of orthogonal space time block codes from second order statistics," IEEE Trans. Inf. Theory, vol. 54, no. 2, pp. 709-722, 2008.

[23] A. Hyvarinen, J. Karunen, and E. Oja, Independent Component Analysis. John Wiley and Sons, 2001.

[24] B. Gu, J. Liu, and Y. Yu, "Orthogonal detection of beam space time block coding using ICA," in IEEE Neural Netw. Brain, vol. 2, Beijing, China, Oct. 2005, pp. 836-840.

[25] J. Via, I. Santamaria, and J. Peres, "Blind identification of MIMOOSTBC channels combining second and higher order statistics," in European Signal Process. Conf., EUSIPCO, Florence, Italy, Sep. 2006.

[26] E. Beres and R. Adve, "Blind channel estimation for orthogonal STBC in MISO systems," IEEE Trans. Veh. Technol., vol. 56, no. 4, pp. 20422050, 2007.

[27] S. Daumont and D. L. Guennec, "Blind source separation with order recovery for MIMO system and an Alamouti or Tarokh space-time block coding scheme," in Proc. IEEE International Symp. Signal Process. Inf. Technol., Cairo, Egypt, Dec. 2007, pp. 431-436.

[28] H. Iglesias, J. Garcia-Naya, and A. Dapena, "A blind channel estimation strategy for the $2 \times 1$ Alamouti system based on diagonalising 4th order cumulant matrices," in Proc. International Conf. Acoustic Speech Signal Process., Las Vegas, USA, Mar. 2008, pp. 3329-3332.

[29] A. Mansour, J. Youssef, and K. Yao, "Underdetermined BSS of MISO OSTBC signals," in ICA, ser. Lecture Notes Comput. Science, vol. 5441, Paraty, Brazil, Mar. 2009, pp. 678-685.

[30] H. Xu, J. Liu, A. Perez-Neira, and M. Lagunas, "Independent component analysis applied to multiple antenna space-time systems," in Proc. IEEE 16th International Symp. Personal, Indoor Mobile Radio Commun., Berlin, Germany, 2005, pp. 57-61.

[31] J. Liu, A. Iserte, and M. Lagunas, "Blind separation of OSTBC signals using ICA neural networks," in Proc. IEEE ISSPIT, Darmstadt, Germany, Dec. 2003, pp. 502-505.

[32] E. Larsson and P. Stoica, Space-Time Block Coding for Wireless Communication. Cambridge University Press, 2003.

[33] M. Shi, Y. Bar-Ness, and W. Su, "STC and BLAST MIMO modulation recognition," in Proc. IEEE GLOBECOM, Nov. 2007, pp. 3034-3039.

[34] V. Choqueuse, K. Yao, L. Collin, and G. Burel, "Blind recognition of linear space time block codes," in Proc. IEEE ICASSP, Las Vegas, USA, Mar. 2008, pp. 2833-2836.

[35] — - "Hierarchical space time block code recognition using correlation matrices," IEEE Trans. Wireless Commun., vol. 7, no. 9, pp. 3526-3534, 2008.

[36] V. Choqueuse, M. Marazin, L. Collin, K. Yao, and G. Burel, "Blind recognition of linear space-time block codes: a likelihood-based approach," IEEE Trans. Signal Process., vol. 58, no. 3, pp. 1290-1299, 2010.

[37] J. Brewer, "Kronecker products and matrix calculus in system theory," IEEE Trans. Circuits Syst., vol. 25, no. 9, pp. 772-781, 1978.

[38] A. Swami and B. Sadler, "Hierarchical digital modulation classification using cumulants," IEEE Trans. Commun., vol. 48, no. 3, pp. 416-429, 2000 .
[39] N. Delfosse and P. Loubaton, "Adaptive separation of independent sources: a deflation approach," in Proc. IEEE International Conf. Acoustic, Speech Signal Process., Adelaide, Australia, 1994, pp. 41-44.

[40] J. Cardoso and B. Laheld, "Equivariant adaptive source separation," IEEE Trans. Signal Process., vol. 44, no. 12, pp. 3017-3030, 1996.

[41] E. Moreau and O. Macchi, "High order contrasts for self-adaptive source separation," International J. Adaptive Control Signal Process., vol. 10, no. 1, pp. 19-46, 1996.

[42] E. Moreau, "Criteria for complex sources separation," in Proc. EUSIPCO, Trieste, Italy, 1996, pp. 931-934.

[43] H. Li and T. Adali, "A class of complex ICA algorithms based on the kurtosis cost function," IEEE Trans. Neural Netw., vol. 19, no. 3, pp. 408-420, 2008

[44] D. Brandwood, "A complex gradient operator and its application in adaptive array theory," IEE Process., Parts $F$ and $H$, vol. 130, no. 1, pp. 11-16, 1983.

[45] L'Ecuyer, "Note: on the interchange of derivative and expectation for likelihood ratio derivative estimators," Management Science, vol. 41, no. 4, pp. 738-747, 1995.

[46] C. Papadias, "Globally convergent blind source separation based on a multiuser kurtosis maximisation criterion," IEEE Trans. Signal Process., vol. 48, no. 12, pp. 3508-3519, 2000.

[47] T. Abrudan, "Advanced optimization algorithms for sensor arrays and multi-antenna communications," Ph.D. dissertation, Helsinky University of Technology, 2008.

[48] T. Abrudan, J. Eriksson, and V. Koivunen, "Steepest descent algorithms for optimization under unitary matrix constraint," IEEE Trans. Signal Process., vol. 56, no. 3, pp. 1134-1147, 2008.

[49] J. Manton, "Optimization algorithms exploiting unitary constraints," IEEE Trans. Signal Process., vol. 50, no. 3, pp. 635-650, 2004.

[50] E. Polak, Optimization: Algorithms and Consistent Approximations. Springer-Verlag, 1997.

[51] A. Mansour and M. Kawamoto, "ICA papers classified according to their applications and performances," IEICE Trans. Fundamentals Electron., Commun. Comput. Sciences, vol. E86, no. A, pp. 620-633, 2000.

[52] "IEEE 802.16e, standard for local and metropolitan area networks, part 16: air interface for fixed and mobile broadband wireless access system," 2005.

[53] B. Vucetic and J. Yuan, Space-Time Coding. John Wiley \& Sons, 2003.

[54] J. Cardoso and A. Soloumiac, "Blind beamforming for non-Gaussian signals," in IEE Proc. F, vol. 140, no. 46, pp. 362-370, 1993.

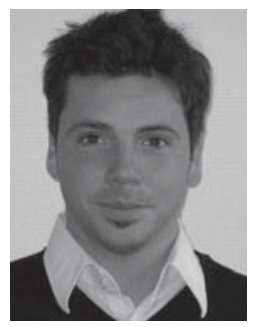

Vincent Choqueuse (S'08-M'09) was born in 1981 in Brest, France. He received the Dipl.-Ing. and the M.Sc. degrees in 2004 and 2005, respectively, from Troyes University of Technology (UTT), France, and the Ph.D. degree in 2008 from University of Brest, France. Since September 2009, he has been Associate Professor at the IUT of Brest, France, and a member of the Laboratory LBMS (EA 4325). His research interests focus on signal processing and statistics for communications and diagnosis.

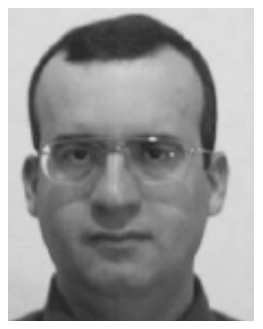

Ali Mansour (M'97,SM'00) was born at Tripoli in Lebanon in 1969. He received his ElectronicElectrical Engineering Diploma in 1992 from the Lebanese University, Tripoli, Lebanon, and his M.Sc. and the Ph.D. degrees in Signal, Image and Speech Processing from INPG, Grenoble, France, in 1993 and 1997, respectively. From January 1997 to July 1997, he held a post doc position at LTIRFINPG, Grenoble, France. From August 1997 to September 2001, he was a Research Scientist at the Bio-Mimetic Control Research Center of Riken, Nagoya, Japan. From 2001 to 2008, he was holding a teacher-researcher position at the Ecole Nationale Supérieure des Ingénieurs des Etudes et Techniques d'Armement (ENSIETA),Brest, France. Since February 2008, he has been a senior-lecturer at the Department of Electrical and Computer Engineering at Curtin University of Technology, Perth, Australia. His research interests are in the areas of blind separation of sources, high order statistics, signal processing, COMINT, radar, sonar and robotics. 


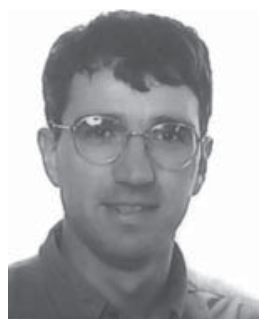

Ludovic Collin received the Ph.D. degree in electrical engineering from the University of Bretagne Occidentale, Brest, France, in 2002. From 1989 to 1999 he was with ORCA Instrumentation, Brest, where he developed oceanographical instrumentation and acoustic modems. From 1999 to 2002 he was Research and Teaching Assistant at French Naval Academy, Lanveoc, France, and at the Institute of Technology of Lannion. From 2003 to 2007 he was Assistant Professor at the ENSIETA, Brest. Since 2007 he has been Assistant Professor at the University of Brest and member of the Laboratory for Science and Technologies of Information, Communication and Knowledge (Lab-STICC - UMR CNRS 3192). His research interests are in MIMO systems and interception of communications.

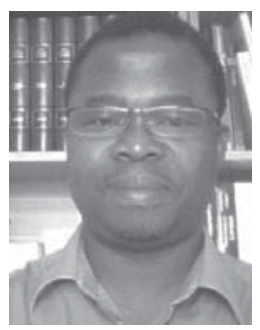

Koffi Clément Yao (M’05) received the PhD degree in Optical Signal Processing and computer sciences from University Louis Pasteur of Strasbourg, France in 1990. After his post-doctorate research on optical neural networks at Ecole Nationale Supérieure des Télécommunications of Brest, he joined the French naval academy as assistant professor in statistical signal processing in 1992. His research interest was focused on Pattern recognition and blind signal separation in underwater acoustics. Since 2001, he has been Assistant Professor at University of Brest,

France. His present research interests are in MIMO systems and blind interception of digital communication signals.

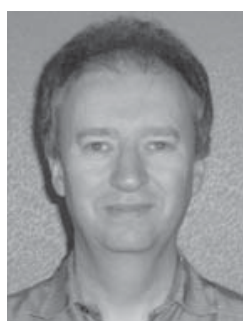

Gilles Burel (M'00-SM'08) was born in 1964. He received the M.Sc. degree from Ecole Supérieure d'Electricité, Gif Sur Yvette, France, in 1988, the Ph.D. degree from University of Brest, France, 1991, and the Habilitation to Supervise Research degree in 1996. From 1988 to 1997 he was a member of the technical staff of Thomson CSF, then Thomson Multimedia, Rennes, France, where he worked on image processing and pattern recognition applications as project manager.

Since 1997, he has been Professor of Digital Communications, Image and Signal Processing at the University of Brest. $\mathrm{He}$ is Associate Director of the Laboratory for Science and Technologies of Information, Communication and Knowledge (Lab-STICC - UMR CNRS $3192)$. He is author or co-author of 19 patents, one book and 140 scientific papers. His present research interests are in signal processing for digital communications, MIMO systems and interception of communications. 